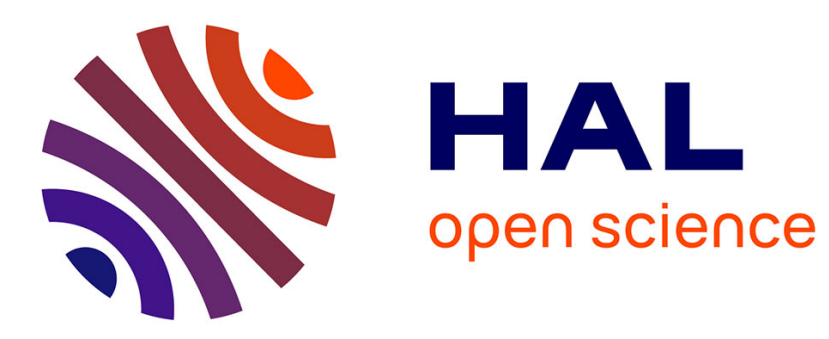

\title{
Absolute Convergence of Rational Series is Semi-decidable
}

Raphaël Bailly, François Denis

\section{To cite this version:}

Raphaël Bailly, François Denis. Absolute Convergence of Rational Series is Semi-decidable. LATA 2009, Apr 2009, Tarragona, Spain. pp.117-128. hal-00359263

\section{HAL Id: hal-00359263 https://hal.science/hal-00359263}

Submitted on 17 Mar 2009

HAL is a multi-disciplinary open access archive for the deposit and dissemination of scientific research documents, whether they are published or not. The documents may come from teaching and research institutions in France or abroad, or from public or private research centers.
L'archive ouverte pluridisciplinaire HAL, est destinée au dépôt et à la diffusion de documents scientifiques de niveau recherche, publiés ou non, émanant des établissements d'enseignement et de recherche français ou étrangers, des laboratoires publics ou privés. 


\title{
Absolute Convergence of Rational Series is Semi-Decidable
}

\author{
Raphaël Bailly, François Denis ${ }^{\star}$ \\ Laboratoire d'Informatique Fondamentale de Marseille \\ CNRS, Aix-Marseille Université \\ $\{$ raphael.bailly, francois.denis $\}$ @lif .univ-mrs.fr
}

\begin{abstract}
We study real-valued absolutely convergent rational series, i.e. functions $r: \Sigma^{*} \rightarrow \mathbb{R}$, defined over a free monoid $\Sigma^{*}$, that can be computed by a multiplicity automaton $A$ and such that $\sum_{w \in \Sigma^{*}}|r(w)|<\infty$. We prove that any absolutely convergent rational series $r$ can be computed by a multiplicity automaton $A$ which has the property that $r_{|A|}$ is simply convergent, where $r_{|A|}$ is the series computed by the automaton $|A|$ derived from $A$ by taking the absolute values of all its parameters. Then, we prove that the set $\mathcal{A}^{\text {rat }}(\Sigma)$ composed of all absolutely convergent rational series is semi-decidable and we show that the sum $\sum_{w \in \Sigma^{*}}|r(w)|$ can be estimated to any accuracy rate for any $r \in \mathcal{A}^{r a t}(\Sigma)$. We also introduce a spectral radius-like parameter $\rho_{|r|}$ which satisfies the following property: $r$ is absolutely convergent iff $\rho_{|r|}<1$.
\end{abstract}

\section{Introduction}

Given a finite alphabet $\Sigma$, we consider real formal power series defined over the free monoid $\Sigma^{*}$, i.e. functions which map $\Sigma^{*}$ into $\mathbb{R}$. More precisely, we consider rational series, which admit several characterizations, one of which being that they can be computed by multiplicity automata $[1,2]$. Given a rational series $r: \Sigma^{*} \rightarrow \mathbb{R}$, we study whether $r$ is absolutely convergent, i.e. $\sum_{w \in \Sigma^{*}}|r(w)|<\infty$. It is polynomially decidable whether a rational series $r$ is simply convergent, i.e. whether the sum $\sum_{n \geq 0} \sum_{w \in \Sigma^{n}} r(w)$ converges to a limit (see [3] for example). Since the Hadamard product $r$ of two rational series $s$ and $t$, defined by $r(w)=$ $s(w) t(w)$, is rational, it is polynomially decidable whether a rational series $r$ converges in quadratic norm, i.e. $\sum_{w \in \Sigma^{*}} r^{2}(w)<\infty$. However, to our knowledge, it is still unknown whether it can be decided if a rational series is absolutely convergent.

The motivation for the present work comes from a problem we have studied in grammatical inference. A stochastic language over $\Sigma^{*}$ is a series $p$ which takes only non negative values and s.t. $\sum_{w \in \Sigma^{*}} p(w)=1^{1}$. A classical problem in grammatical inference consists in inferring an estimate of a target stochastic language $p$ from a finite sample of words $\left\{w_{1}, \ldots, w_{n}\right\}$ independently drawn

\footnotetext{
* This research was partially supported by the ANR project Marmota

1 This definition differs from the one given in [2]
} 
according to $p$. In [4], we proposed an algorithm DEES which takes a sample as input and outputs a rational series $r$ which simply converges to 1 but can take negative values, and which satisfies the following property: with probability one, there exists a sample size level from which the sum $\sum_{w \in \Sigma^{*}}|p(w)-r(w)|$ is arbitrarily small, (which implies that $r$ is absolutely convergent); moreover, a stochastic language $p_{r}$ can be computed from $r$, that satisfies

$$
\sum_{w \in \Sigma^{*}}\left|p_{r}(w)-r(w)\right| \leq \sum_{w \in \Sigma^{*}}|r|(w)-1 .
$$

In other words, we know that from some sample size, we will have a solution of our problem. But we need to decide whether the series $r$ output by DEES from the working sample is absolutely convergent to ensure that $r$ provides a solution and we need to compute an estimate of $\sum_{w \in \Sigma^{*}}|r|(w)$ to bound the accuracy of this solution.

A multiplicity automaton (MA) is a tuple $A=\langle\Sigma, Q, \varphi, \iota, \tau\rangle$, where $Q$ is a finite set of states, $\varphi$ (resp. $\iota, \tau)$ is a transition (resp. initialization, termination) function, which can be used to compute a rational series $r_{A}$. Any rational series $r$ can be computed by a MA. Given a MA $A$, we obtain a new MA $|A|$ by taking the absolute values of the functions $\varphi, \iota$ and $\tau$. It is straightforward that $r_{A}$ is absolutely convergent if $r_{|A|}$ is simply convergent. We prove that any absolutely convergent rational series $r$ can be computed by a multiplicity automaton $A$ which has the property that $r_{|A|}$ is simply convergent. Then, we provide an algorithm which takes a multiplicity automaton $B$ as input and halts if and only if $r_{B}$ is absolutely convergent: when the algorithm halts, it outputs a MA $A$ equivalent to $B$, i.e. the series computed by $A$ and $B$ are equal, and such that $r_{|A|}$ is convergent. So, we have proved that the set $\mathcal{A}^{\text {rat }}(\Sigma)$ composed of all absolutely convergent rational series is semi-decidable.

The sum $\sum_{w \in \Sigma^{*}}|r(w)|$ can be estimated from below by computing the sum $\sum_{w \in \Sigma \leq n}|r(w)|$ for increasing integers $n$. We prove that our algorithm can be used to provide convergent upper bounds. So, the sum $\sum_{w \in \Sigma^{*}}|r(w)|$ can be estimated to any accuracy rate. As a consequence, if the L1-distance $\|r-s\|_{1}=\sum_{w \in \Sigma^{*}}|r(w)-s(w)|$ is finite, it can be estimated to any accuracy rate. It has been proved in [5] that computing the $L 1$-distance between two hidden Markov models is $N P$-hard which implies that computing the $L 1$-distance between two MA is $N P$-hard too. $L_{p}$ distances of two probabilistic automata have been studied in $[6,7]$, where efficient algorithm have been provided when $p$ is even. Our algorithm can be used to estimate $L_{p}$ distances between two rational series for any odd values of $p$.

Finally, for any rational series $r$, we introduce a spectral radius-like parameter, $\rho_{|r|}$ defined by $\rho_{|r|}=\limsup _{n}\left(|r|\left(\Sigma^{n}\right)\right)^{1 / n}$ and we show that $r$ is absolutely convergent iff $\rho_{|r|}<1$.

We recall some properties on rational series and multiplicity automata in Section 2. We study absolutely convergent series in Section 3; in particular, we prove that any absolutely convergent rational series can be represented by a MA $A$ such that $r_{|A|}$ is convergent. We prove the semi-decidability of the class 
$\mathcal{A}^{\text {rat }}(\Sigma)$ in Section 4 . We show how the sum $\sum_{w \in \Sigma^{*}}|r(w)|$ can be estimated in Section 5 . To conclude, we provide some comments and describe some conjectures and future works in Section 6.

\section{Preliminaries}

\subsection{Rational Series}

Let $\Sigma^{*}$ be the set of words on the finite alphabet $\Sigma$. The empty word is denoted by $\varepsilon$, and the length of a word $u$ is denoted by $|u|$. For any integer $k$, we denote by $\Sigma^{k}$ the set $\left\{u \in \Sigma^{*}|| u \mid=k\right\}$ and by $\Sigma^{\leq k}$ the set $\left\{u \in \Sigma^{*}|| u \mid \leq k\right\}$. A subset $S$ of $\Sigma^{*}$ is prefix-closed if for any $u, v \in \Sigma^{*} u v \in S \Rightarrow u \in S$.

The general context is, for an alphabet $\Sigma$ the set $\mathbb{R}\langle\langle\Sigma\rangle\rangle$ of all the mappings from $\Sigma^{*}$ into $\mathbb{R}$. An element of this set is called a formal power series. This set is an $\mathbb{R}$-vector space. For any series $r$ and any word $u \in \Sigma^{*}$, we denote by $u r$ the series defined by $\dot{u} r(w)=r(u w)$. Let $L H(r)$ denote the linear hull of $r$, i.e. the vector subspace of $\mathbb{R}\langle\langle\Sigma\rangle\rangle$ spanned by $\left\{\dot{u} r \mid u \in \Sigma^{*}\right\}$.

A multiplicity automaton $(M A)$ is a tuple $\langle\Sigma, Q, \varphi, \iota, \tau\rangle$ where $Q$ is a finite set of states, $\varphi: Q \times \Sigma \times Q \rightarrow \mathbb{R}$ is the transition function, $\iota: Q \rightarrow \mathbb{R}$ is the initialization function and $\tau: Q \rightarrow \mathbb{R}$ is the termination function. Let $Q_{I}=\{q \in Q \mid \iota(q) \neq 0\}$ be the set of initial states and $Q_{T}=\{q \in Q \mid \tau(q) \neq 0\}$ be the set of terminal states. We extend the transition function $\varphi$ to $Q \times \Sigma^{*} \times Q$ by $\varphi\left(q, w x, q^{\prime}\right)=\sum_{q^{\prime \prime} \in Q} \varphi\left(q, w, q^{\prime \prime}\right) \varphi\left(q^{\prime \prime}, x, q^{\prime}\right)$ and $\varphi\left(q, \varepsilon, q^{\prime}\right)=1$ if $q=q^{\prime}$ and 0 otherwise, for any $q, q^{\prime} \in Q, x \in \Sigma$ and $w \in \Sigma^{*}$. For any finite subset $L \subset \Sigma^{*}$ and any $Q^{\prime} \subseteq Q$, define $\varphi\left(q, L, Q^{\prime}\right)=\sum_{w \in L, q^{\prime} \in Q^{\prime}} \varphi\left(q, w, q^{\prime}\right)$. For any MA $A=$ $\langle\Sigma, Q, \varphi, \iota, \tau\rangle$, we define the series $r_{A}$ by $r_{A}(w)=\sum_{q, q^{\prime} \in Q} \iota(q) \varphi\left(q, w, q^{\prime}\right) \tau\left(q^{\prime}\right)$. For any $q \in Q$, we define the series $r_{A, q}$ by $r_{A, q}(w)=\sum_{q^{\prime} \in Q} \varphi\left(q, w, q^{\prime}\right) \tau\left(q^{\prime}\right)$. The support of a MA $\langle\Sigma, Q, \varphi, \iota, \tau\rangle$ is a non deterministic finite automaton (NFA) $\left\langle\Sigma, Q, \delta, Q_{I}, Q_{F}\right\rangle$ where the transition function is defined by $\delta(q, x)=\left\{q^{\prime} \in\right.$ $\left.Q \mid \varphi\left(q, x, q^{\prime}\right) \neq 0\right\}$.

We will say that a series $r$ is rational if it satisfies one of the two following equivalent conditions:

1. the dimension of $L H(r)$ is finite;

2. $r$ can be computed by a multiplicity automaton.

The family of all rational series is denoted by $\mathbb{R}^{r a t}\langle\langle\Sigma\rangle\rangle$.

\subsection{Prefixial Multiplicity Automata}

Representation of rational series based on prefix sets has been introduced in [1].

Definition 1. Let $A=\langle\Sigma, Q, \varphi, \iota, \tau\rangle$ be a $M A$. We say that $A$ is prefixial if:

- $Q$ is non-empty prefix-closed finite subset of $\Sigma^{*}$

$-\forall u \in Q, \iota(u) \neq 0$ iff $u=\varepsilon$

$-\forall x \in \Sigma, \forall u, v \in Q$ s.t. $u x \in Q, \varphi(u, x, v) \neq 0$ iff $v=u x$. 
A transition $(u, x, v)$ is called an inner transition if $v=u x$ and a border transition otherwise. The set $F=\{u x \mid u \in Q, x \in \Sigma, u x \notin Q\}$ is called the frontier set of $A$.

An NFA $A=\langle\Sigma, Q, \delta, I, F\rangle$ is prefixial if $A$ is the support of a prefixial MA.

A prefix-closed subset $Q$ of $\Sigma^{*}$ can be used as the set of states of a prefixial automaton that computes a rational series $r$ if and only if the set $\{\dot{u} r \mid u \in Q\}$ spans $L H(r)$. Let us make this statement precise.

Let $r$ be a rational series and let $Q$ be a prefix-closed subset such that $\{\dot{u} r \mid u \in$ $Q\}$ spans $L H(r)$. Let $F=\{u x \mid u \in Q, x \in \Sigma, u x \notin Q\}$. Let $f: Q \rightarrow \mathbb{R}$ and $g: F \times Q \rightarrow \mathbb{R}$ be two mappings that satisfy:

1. $f(u) \neq 0$ for every state $u$,

2. $\dot{\overline{u x}} r=\sum_{v \in Q} g(u x, v) \frac{\bar{f}(u)}{\bar{f}(v)} \dot{v} r$ for every $u x \in F$ where $\bar{f}$ is defined by $\bar{f}(\varepsilon)=$ $f(\varepsilon)$, where $\bar{f}(w x)=\bar{f}(w) f(w x)$ for any $w \in \Sigma^{*}$ and $x \in \Sigma$ and where the top bar notation is meant to express that the "dot" applies to the element under the bar. The function $g$ expresses linear dependencies.

We define the prefixial automaton $A(\Sigma, Q, f, g, r)=\langle\Sigma, Q, \varphi, \iota, \tau\rangle$ by

$-\iota(\varepsilon)=f(\varepsilon)$,

- $\forall u \in Q, x \in \Sigma$ s.t. $u x \in Q, \varphi(u, x, u x)=f(u x)$,

- $\forall u, v \in Q, x \in \Sigma$ s.t. $u x \in F, \varphi(u, x, v)=g(u x, v)$,

$-\forall u \in Q, \tau(u)=\frac{r(u)}{\bar{f}(u)}$.

Proposition 1. The automaton $A(\Sigma, Q, f, g, r)$ computes $r$.

Proof. Let $A(\Sigma, Q, f, g, r)=\langle\Sigma, Q, \varphi, \iota, \tau\rangle$. Let us show, by induction on $|w|$, that for any $u \in Q$ and any $w \in \Sigma^{*}, \dot{u} r(w)=\bar{f}(u) r_{A, u}(w)$.

- Let $u \in Q: \dot{u} r(\varepsilon)=r(u)$ and $\bar{f}(u) r_{A, u}(\varepsilon)=\bar{f}(u) \tau(u)=r(u)$.

- Let $u \in Q, w \in \Sigma^{*}$ and $x \in \Sigma$.

$$
\text { If } u x \in Q, \quad \begin{aligned}
\bar{f}(u) r_{A, u}(x w) & =\bar{f}(u) \varphi(u, x, u x) r_{A, u x}(w) \\
& =\bar{f}(u x) r_{A, u x}(w) \text { by definition of } \bar{f} \\
& =\bar{f}(u x) \frac{\overline{u x} r(w)}{\bar{f}(u x)} \text { by induction hypothesis } \\
& =\overline{u x} r(w) .
\end{aligned}
$$

If $u x \notin Q, \bar{f}(u) r_{A, u}(x w)=\bar{f}(u) \sum_{v \in Q} g(u x, v) r_{A, v}(w)$ by definition of $A$

$$
=\bar{f}(u) \sum_{v \in Q} g(u x, v) \frac{\dot{v} r(w)}{\bar{f}(v)} \text { by induction hypothesis }
$$

$=\dot{\overline{u x}} r(w)$ by definition of $g$.

Therefore, for any $u \in Q, \dot{u} r=\bar{f}(u) r_{A, u}$ and in particular, $r=f(\varepsilon) r_{A, \varepsilon}=r_{A}$. 


\section{On Representation of Absolutely Convergent Rational Series}

\subsection{Absolutely Convergent Rational Series}

Let $r$ be a series and let $\Gamma$ be a non-empty subset of $\Sigma^{*}$. We say that $r$ is convergent on $\Gamma$ if the sum $\sum_{n>0} \sum_{w \in \Gamma \cap \Sigma^{n}} r(w)$ is convergent; if so, we denote the sum by $r(\Gamma)$. Let $|r|$ be the series defined by $|r|(w)=|r(w)|$. We say that $r$ is absolutely convergent if $|r|$ is convergent. Note that when a series $r$ is absolutely convergent, it is convergent over any $\Gamma \subseteq \Sigma^{*}$.

We denote by $\mathcal{A}(\Sigma)$ (resp. by $\mathcal{A}^{\text {rat }}(\Sigma)$ ) the subspace of $\mathbb{R}\langle\langle\Sigma\rangle\rangle$ (resp. of $\left.\mathbb{R}^{\text {rat }}\langle\langle\Sigma\rangle\rangle\right)$ composed of the series that are absolutely convergent.

Let $r$ be a series, we denote by $\operatorname{res}(r)$ the following subset of $\Sigma^{*}$ : res $(r)=$ $\left\{u \in \Sigma^{*} / \exists w \in \Sigma^{*}, r(u w) \neq 0\right\}$. For any absolutely convergent series $r$ and any word $u \in \operatorname{res}(r)$, we denote by $u^{-1} r$ the series defined by $u^{-1} r(w)=\frac{|r|\left(\Sigma^{*}\right)}{|r|\left(u \Sigma^{*}\right)} \dot{u} r$ and we call it the residual of $r$ associated with $u$. The set of all the residuals of $r$ is denoted by $\operatorname{Res}(r)$. The vector subspace spanned by $\operatorname{Res}(r)$ is equal to $L H(r)$. Note that for any $u \in \operatorname{res}(r),\left|u^{-1} r\right|\left(\Sigma^{*}\right)=|r|\left(\Sigma^{*}\right)$. The mapping $r \rightarrow|r|\left(\Sigma^{*}\right)$ defines a norm $\|\cdot\|_{1}$ on $\mathcal{A}(\Sigma)$. Let us denote by $C H(r)$ the convex hull of Res $(r)$ : $C H(r)=\left\{\sum_{i=1}^{n} \alpha_{i} u_{i}^{-1} r \mid n \in \mathbb{N}, \alpha_{i} \geq 0, \sum_{i=1}^{n} \alpha_{i}=1, u_{i} \in \operatorname{res}(r)\right\}$. Let us denote by $C C H(r)$, the closed convex hull of Res(r), i.e. the closure of $C H(r)$. Note that when $r \in \mathcal{A}^{r a t}(\Sigma),\|\cdot\|_{1}$ is constant on $C C H(r)$. In particular, $C C H(r)$ is a compact convex set.

Lemma 1. Let $r \in \mathcal{A}^{\text {rat }}(\Sigma)$. Then $\forall \epsilon>0, \exists k \in \mathbb{N}$ such that $\forall s \in C C H(r)$, $|s|\left(\Sigma^{>k}\right)<\epsilon$. In particular, $\forall \epsilon>0, \exists k \in \mathbb{N}$ such that $\forall u \in \operatorname{res}(r),|r|\left(u \Sigma^{>k}\right)<$ $\epsilon|r|\left(u \Sigma^{*}\right)$.

Proof. For any integer $k$, let $f_{k}: C C H(r) \rightarrow \mathbb{R}$ be defined by $f_{k}(s)=|s|\left(\Sigma^{>k}\right)$. For any $s, t \in C C H(r)$, we have $\left|f_{k}(s)-f_{k}(t)\right| \leq\|s-t\|_{1}$. Hence, $f_{k}$ is continuous for any $k$. Moreover, $\lim _{k \rightarrow \infty} f_{k}(s)=0$ for any $s \in C C H(r)$. Since $C C H(r)$ is compact, $\left(f_{k}\right)$ converges uniformy to 0 : for any $\epsilon>0$, there exists $K \geq 0$ s.t. for any $k \geq K$ and any $s \in C C H(r),\left|f_{k}(s)\right|<\epsilon$. Apply the result to $s=u^{-1} r$ and $\epsilon /|r|\left(\Sigma^{*}\right)$ to obtain the second result.

Lemma 2. Let $r \in \mathcal{A}^{\text {rat }}(\Sigma)$ and let $d=\operatorname{dim}(L H(r))$. For all $\epsilon>0$, there exists an integer $N$ such that for any $u \in \operatorname{res}(r)$, there exists $v_{1}, \ldots, v_{d} \in \operatorname{res}(r) \cap \Sigma \leq N$ and $\alpha_{1}, \ldots, \alpha_{d}>-\epsilon$ such that $\sum_{1 \leq i \leq d} \alpha_{i}<1+\epsilon$ and $u^{-1} r=\sum_{1 \leq i \leq d} \alpha_{i} v_{i}^{-1} r$.

Proof. Suppose that there exists $\epsilon>0$ such that for any integer $n$, there exists $u_{n} \in \operatorname{res}(r)$ such that for any $v_{1}, \ldots, v_{d} \in \operatorname{res}(r) \cap \Sigma^{\leq n}, u_{n}^{-1} r=\sum_{i=1}^{d} \alpha_{i} v_{i}^{-1} r$ implies that there exists an index $i$ s.t. $\alpha_{i} \leq-\epsilon$, or $\sum_{i=1}^{d} \alpha_{i} \geq 1+\epsilon$. Let $n_{k}$ be a subsequence such that $u_{n_{k}}^{-1} r$ converges to an absolutely convergent series $s \in C C H(r)$.

Now, let $v_{1}, \ldots, v_{d-1}$ be such that $v_{1}^{-1} r, \ldots, v_{d-1}^{-1} r, s$ form a basis of $L H(r)$. For any integer $k$, let $u_{n_{k}}^{-1} r=\alpha_{1, k} v_{1}^{-1} r+\ldots+\alpha_{d-1, k} v_{d-1}^{-1} r+\alpha_{d, k} s$. Since $u_{n_{k}}^{-1} r$ converges to $s, \alpha_{i, k}$ converges to 0 for $i=1, \ldots, d-1$ and $\alpha_{d, k}$ converges to 1 
when $k$ tends to infinity. Therefore, there should exist an integer $K$ such that for any $k \geq K$, each coefficient of this combination is strictly greater that $-\epsilon$, and the sum of all its coefficient is strictly lower than $1+\epsilon$, which is contradictory.

\subsection{A Particular Representation of Absolutely Convergent Rational Series}

Let $A=\langle\Sigma, Q, \varphi, \iota, \tau\rangle$ be an MA. Let us denote by $|A|$ the MA defined by $|A|=\langle\Sigma, Q,|\varphi|,|\iota|,|\tau|\rangle$.

Lemma 3. $\left|r_{A}\right| \leq r_{|A|}$. Hence, if $r_{|A|}$ is convergent, then $r_{A}$ is absolutely convergent.

Proof. Indeed,

$$
\left|r_{A}(w)\right|=\left|\sum_{q, q^{\prime} \in Q} \iota(q) \varphi\left(q, w, q^{\prime}\right) \tau\left(q^{\prime}\right)\right| \leq \sum_{q, q^{\prime} \in Q}\left|\iota(q) \varphi\left(q, w, q^{\prime}\right) \tau\left(q^{\prime}\right)\right|=r_{|A|}(w) .
$$

Lemma 4. Let $A=\langle\Sigma, Q, \varphi, \iota, \tau\rangle$ be a MA. Suppose that $\varphi$ takes only non negative values and that there exists an integer $k$ such that for any state $q$, $\varphi\left(q, \Sigma^{k}, Q\right)<1$. Then, the series $r_{|A|}$ is convergent.

Proof. Let $R=\sup \left\{\varphi\left(q, \Sigma^{h}, Q\right) \mid q \in Q, h<k\right\}$ and $\rho=\sup \left\{\varphi\left(q, \Sigma^{k}, Q\right) \mid q \in Q\right\}$. From the hypothesis, $\rho<1$.

Since for any state $q$ and any integers $n>0$ and $0 \leq h<k, \varphi\left(q, \Sigma^{n k+h}, Q\right)=$ $\sum_{q^{\prime} \in Q} \varphi\left(q, \Sigma^{(n-1) k+k}, q^{\prime}\right) \varphi\left(q^{\prime}, \Sigma^{h}, Q\right)$, it can easily be shown by induction on $n$ that $\varphi\left(q, \Sigma^{n k+h}, Q\right) \leq R \rho^{n}$.

Let $I=\operatorname{Sup}\{|\iota(q)|$ for $q \in Q\}$ and $T=\operatorname{Sup}\{|\tau(q)|$ for $q \in Q\}$. We have

$\left|r_{A}\right|\left(\Sigma^{n k+h}\right) \leq \sum_{q, q^{\prime} \in Q}|\iota(q)| \varphi\left(q, \Sigma^{n k+h}, q^{\prime}\right)\left|\tau\left(q^{\prime}\right)\right| \leq I T \sum_{q \in Q} \varphi\left(q, \Sigma^{n k+h}, Q\right) \leq I T R|Q| \rho^{n}$

Therefore, $\left|r_{A}\right|\left(\Sigma^{*}\right)=\sum_{n \geq 0} \sum_{h=0}^{k-1}\left|r_{A}\right|\left(\Sigma^{n k+h}\right) \leq I T R|Q| k \sum_{h \geq 0} \rho^{n}=\frac{I T R|Q| k}{1-\rho}$.

Note that if $A$ is prefixial, there exists a unique state $q$ such that $\iota(q) \neq 0$. So we can take $R=\sup \left\{\varphi\left(\varepsilon, \Sigma^{h}, Q\right) \mid q \in Q, h<k\right\}$ and we have $\left|r_{A}\right|\left(\Sigma^{n k+h}\right) \leq \operatorname{ITR} \rho^{n}$. and $\left|r_{A}\right|\left(\Sigma^{*}\right) \leq \frac{I T R k}{1-\rho}$.

Theorem 1. Let $r \in \mathcal{A}^{\text {rat }}(\Sigma)$. Let $\rho<1$. There exists an integer $n$, and a prefixial $M A A=\langle\Sigma, Q, \varphi, \iota, \tau\rangle$ that computes $r$ and such that $\forall u \in Q$, $|\varphi|\left(u, \Sigma^{n}, Q\right)<\rho$. Hence, the series $r_{|A|}$ is convergent. 


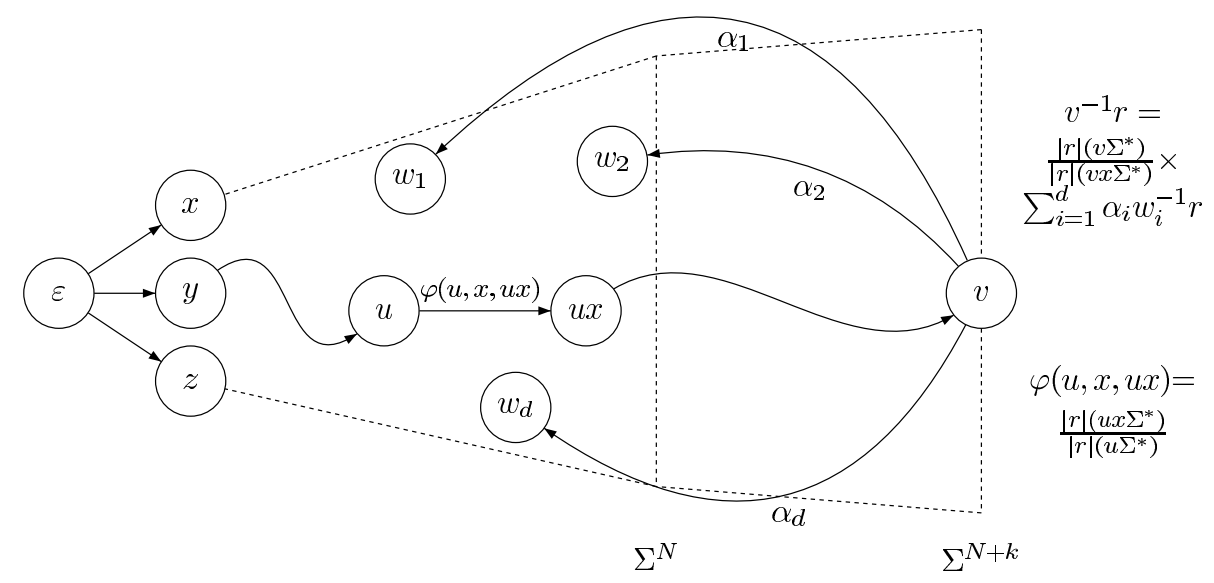

Fig. 1. Automaton built in the proof of Theorem 1

Proof. Let $r \in \mathcal{A}^{\text {rat }}(\Sigma)$ and let $d=\operatorname{dim}(\operatorname{LH}(r))$. Let $\rho<1$ and let $\varepsilon=\rho / 16$. From Lemma 1 , let $k$ be an integer such that $\forall u \in \operatorname{res}(r), \frac{|r|\left(u \Sigma^{\geq k}\right)}{|r|\left(u \Sigma^{*}\right)}<\epsilon$. Let $\epsilon^{\prime}=(d|\Sigma|)^{-1}$. From Lemma 2, let $N>k$ be an integer such that for all $u \in$ $\operatorname{res}(r)$, there exists $v_{u, 1}, \ldots, v_{u, d} \in \operatorname{res}(r) \cap \Sigma^{\leq N}$ and $\alpha_{u, 1}, \ldots, \alpha_{u, d}>-\epsilon^{\prime}$ such that $\sum_{i=1}^{d} \alpha_{u, i}<1+\epsilon^{\prime} \leq 2$ and $u^{-1} r=\sum_{i=1}^{d} \alpha_{u, i} v_{u, i}^{-1} r$.

Let $Q=\Sigma^{N+k} \cap \operatorname{res}(r)$. Let $f: Q \rightarrow \mathbb{R}$ be defined by $f(\varepsilon)=1$ and $\forall u x \in$ $Q, f(u x)=\frac{|r|\left(u x \Sigma^{*}\right)}{|r|\left(u \Sigma^{*}\right)}$ where $u \in \Sigma^{*}$ and $x \in \Sigma$. One can easily show that $\bar{f}(u)=|r|\left(u \Sigma^{*}\right) /|r|\left(\Sigma^{*}\right)$.

Let $F=\{u x \mid u \in Q, x \in \Sigma, u x \notin Q\}$. For any $u x \in F \cap \operatorname{res}(r)$, there exist coefficients $\left(\alpha_{u x, v}\right)_{v \in \Sigma \leq N}$ such that (i) at most $d$ coefficients $\alpha_{u x, v}$ are not null; (ii) $(u x)^{-1} r=\sum_{v \in \Sigma \leq N} \alpha_{u x, v} v^{-1} r$; (iii) $\alpha_{u x, v}>-\epsilon^{\prime}$; (iv) $\sum_{v \in \Sigma \leq N} \alpha_{u x, v}<1+\epsilon^{\prime}$. From $(u x)^{-1} r=\sum_{v \in \Sigma \leq N} \alpha_{u x, v} v^{-1} r$, then $\overline{u x} r=|r|\left(u x \Sigma^{*}\right) \sum_{v \in \Sigma \leq N} \frac{\alpha_{u x, v}}{|r|\left(v \Sigma^{*}\right)} \dot{v} r$.

Let $g: Q \times F \rightarrow \mathbb{R}$ be defined by $g(u x, v)=\alpha_{u x, v} \frac{|r|\left(u x \Sigma^{*}\right)}{|r|\left(u \Sigma^{*}\right)}$ if $u x \in F \cap$ res $(r)$ and 0 otherwise. One can check that the conditions on $f$ and $g$ stated in Section 2.2 are satisfied. (see Fig. 1)

From the proposition 1, the MA $A(\Sigma, Q, f, g, r)=\langle\Sigma, Q, \varphi, \iota, \tau\rangle$ computes $r$. Let us list below some properties of $A$ :

1. $\forall u \in Q \cap \Sigma^{<N+k}$ and $x \in \Sigma, \varphi(u, x, u x)=\frac{|r|\left(u x \Sigma^{*}\right)}{|r|\left(u \Sigma^{*}\right)} \leq 1$;

2. $\forall u \in Q \cap \Sigma^{<N+k}$ and $h<N+k-|u|, \varphi\left(u, \Sigma^{h}, Q\right)=\frac{|r|\left(u \Sigma^{\geq h}\right)}{|r|\left(u \Sigma^{*}\right)} \leq 1$;

3. $\forall u \in Q \cap \Sigma^{\leq N}, \varphi\left(u, \Sigma^{k}, Q\right)=\frac{|r|\left(u \Sigma^{\geq k}\right)}{|r|\left(u \Sigma^{*}\right)}<\varepsilon$;

4. $\forall u \in Q \cap \Sigma^{N+k}$ and $x \in \Sigma, \varphi(u, x, v)=\alpha_{u x, v} \frac{|r|\left(u x \Sigma^{*}\right)}{|r|\left(u \Sigma^{*}\right)}>-\varepsilon^{\prime}$;

5. $\forall u \in Q \cap \Sigma^{N+k}, \varphi(u, \Sigma, Q)<1+\varepsilon^{\prime} \leq 2$;

6. $\forall u \in \Sigma^{N+k},(|\varphi|(u, \Sigma, Q)-\varphi(u, \Sigma, Q))<2 d|\Sigma| \epsilon^{\prime}$.

From these properties, one can deduce that: 
a) For all $u \in \Sigma^{h}, h \leq N-k$ one have

$$
|\varphi|\left(u, \Sigma^{2 k}, Q\right)=\sum_{w \in \Sigma^{k}} \varphi(u, w, u w) \varphi\left(u w, \Sigma^{k}, Q\right)<\epsilon^{2}<\rho
$$

by applying twice the property 3 .

b) For $u \in \Sigma^{N-k+1}$ one have

$$
|\varphi|\left(u, \Sigma^{2 k}, Q\right)<\epsilon\left(2+2 d|\Sigma| \epsilon^{\prime}\right)<\rho .
$$

Indeed, $|\varphi|\left(u, \Sigma^{2 k}, Q\right)=$

$$
\begin{aligned}
& \sum_{|w|=2 k-1} \varphi(u, w, u w)[\varphi(u w, \Sigma, Q)+(|\varphi|(u w, \Sigma, Q)-\varphi(u w, \Sigma, Q))] \\
\leq & \sum_{|w|=2 k-1} \varphi(u, w, u w)\left[2+2 d|\Sigma| \epsilon^{\prime}\right] \text { from properties } 5 \text { and } 6 \\
\leq & \varepsilon\left(2+2 d|\Sigma| \epsilon^{\prime}\right) \text { from properties } 2 \text { and } 3 .
\end{aligned}
$$

c) For $u \in \Sigma^{h}$ where $N-k+1<h \leq N$ one have

$$
|\varphi|\left(u, \Sigma^{2 k}, Q\right)<\epsilon\left(2+2 d|\Sigma| \epsilon^{\prime}\right)<\rho .
$$

Indeed, $|\varphi|\left(u, \Sigma^{2 k}, Q\right)=$

$$
\begin{aligned}
& \sum_{|w|=N+k-h} \varphi(u, w, u w) \sum_{|v| \leq N}|\varphi|(u w, \Sigma, v) \varphi\left(v, \Sigma^{k+h-N-1}, Q\right) \\
\leq & \sum_{|w|=N+k-h} \varphi(u, w, u w) \sum_{|v| \leq N}|\varphi|(u w, \Sigma, v) \text { from property } 2 \\
\leq & \sum_{|w|=N+k-h} \varphi(u, w, u w)[\varphi(u w, \Sigma, Q)+(|\varphi|(u w, \Sigma, Q)-\varphi(u w, \Sigma, Q))] \\
\leq & \sum_{|w|=N+k-h} \varphi(u, w, u w)\left[2+2 d|\Sigma| \epsilon^{\prime}\right] \text { from properties } 5 \text { and } 6 \\
\leq & \varepsilon\left(2+2 d|\Sigma| \epsilon^{\prime}\right) \text { from properties } 2 \text { and } 3 .
\end{aligned}
$$

d) For $u \in \Sigma^{h}, N<h \leq N+k$ one have

$$
|\varphi|\left(u, \Sigma^{2 k}, Q\right)<\varepsilon\left(2+2 d|\Sigma| \epsilon^{\prime}\right)^{2}<\rho .
$$

Indeed,

$$
|\varphi|\left(u, \Sigma^{2 k}, Q\right)<\sum_{w \in \Sigma^{N+k-h}} \varphi(u, w, u w) \sum_{v \in \Sigma \leq N}|\varphi|(u w, \Sigma, v)|\varphi|\left(v, \Sigma^{h+k-N-1}, Q\right) .
$$




$$
\begin{aligned}
& \text { If }|v| \leq 2 N-h+1,|v|+h+k-N-1 \leq N+k \text {, and from Property } 3, \\
& \qquad|\varphi|\left(v, \Sigma^{h+k-N-1}, Q\right) \leq \epsilon \\
& \text { If }|v|>2 N-h+1,|\varphi|\left(v, \Sigma^{h+k-N-1}, Q\right) \\
& \quad \leq \sum_{|w|=N+k-|v|} \varphi(v, w, v w) \sum_{v^{\prime} \in \Sigma \leq N}|\varphi|\left(v w, \Sigma, v^{\prime}\right) \varphi\left(v^{\prime}, \Sigma^{|v|+h-2 N-2}, Q\right) \\
& \quad \leq \sum_{|w|=N+k-|v|} \varphi(v, w, v w)|\varphi|(v w, \Sigma, Q) \\
& \quad \leq\left(2+2 d|\Sigma| \epsilon^{\prime}\right) \varphi\left(v, \Sigma^{N+k-|v|}, Q\right) \\
& \quad \leq \varepsilon\left(2+2 d|\Sigma| \epsilon^{\prime}\right) .
\end{aligned}
$$

That is, in all cases, $|\varphi|\left(v, \Sigma^{h+k-N-1}, Q\right) \leq \varepsilon\left(2+2 d|\Sigma| \epsilon^{\prime}\right)$ and

$$
\begin{aligned}
|\varphi|\left(u, \Sigma^{2 k}, Q\right) & <\varepsilon\left(2+2 d|\Sigma| \epsilon^{\prime}\right) \sum_{w \in \Sigma^{N+k-h}} \varphi(u, w, u w) \sum_{v \in \Sigma \leq N}|\varphi|(u w, \Sigma, v) \\
& \leq \varepsilon\left(2+2 d|\Sigma| \epsilon^{\prime}\right)^{2}
\end{aligned}
$$

We have proved that for any $u \in Q,|\varphi|\left(u, \Sigma^{2 k}, Q\right)<\rho$. Hence, from lemma 4 , the series $r_{|A|}$ is convergent.

Spectral radius of a matrix, joint or generalized spectral radius of a set of matrices are tools used to study asymptotic properties of powers or products of matrices. A good introduction on spectral radii can be found in the first chapters of $[8]$.

Definition 2. Let $r \in \mathbb{R}^{r a t}\langle\langle\Sigma\rangle\rangle$. We define the absolute spectral radius

$$
\rho_{|r|}=\lim \sup _{n}\left(|r|\left(\Sigma^{n}\right)\right)^{1 / n}
$$

Proposition 2. Let $r \in \mathbb{R}^{r a t}\langle\langle\Sigma\rangle\rangle . r \in \mathcal{A}^{\text {rat }}(\Sigma)$ if and only if $\rho_{|r|}<1$.

Proof. Let $r \in \mathbb{R}^{r a t}\langle\langle\Sigma\rangle\rangle$. Suppose that $\rho_{|r|}<1$, then there exists $\rho$ s.t. $\rho_{|r|}<$ $\rho<1$ and $n \in \mathbb{N}$ such that $|r|\left(\Sigma^{\geq n}\right)<\sum_{i=n}^{\infty} \rho^{i}$. Thus $r$ is absolutely convergent. Suppose now that $r \in \mathcal{A}^{\text {rat }}(\Sigma)$. By the theorem 1, there exists a prefixial automaton $A=\langle\Sigma, Q, \varphi, \iota, \tau\rangle$ that computes $r, \rho<1$ and an integer $n$ such that for every state $q,\left|\varphi_{A}\right|\left(q, \Sigma^{n}, Q\right)<\rho$. Hence, from Lemma $4,|r|\left(\Sigma^{m}\right)=O\left(\rho^{m / n}\right)$ and therefore, $\rho_{\left|r_{A}\right|} \leq \rho^{1 / n}<1$.

\section{Decidability}

We prove in this section that there exists an algorithm that takes a multiplicity automaton $A$ as input and halts iff $r_{A}$ is absolutely convergent. In other words, the class $\mathcal{A}(\Sigma)$ is semi-decidable. 
Theorem 2. The class $\mathcal{A}^{\text {rat }}(\Sigma)$ is semi-decidable.

Proof. Let $r$ be a rational series and let $A$ be a MA that computes $r$. Queries such as: What is the dimension $d$ of $L H(r)$ ? Does the word $u$ belongs to res $(r)$ ? Given $u_{1}, \ldots, u_{d} \in \operatorname{res}(r)$, is $\left\{\dot{u_{1} r}, \ldots, u_{d} r\right\}$ a basis of $L H(r)$ ? can be answered by using $A$.

We consider the countable class $\mathcal{S}_{r}$ composed of all the prefixial NFA $\langle\Sigma, Q, \delta$, $I, T\rangle$ that satisfy the following properties:

- there exists two integers $N$ and $k$ such that $Q=\Sigma \leq N+k \cap \operatorname{res}(u)$;

- for any $u \in Q$ and $x \in \Sigma$ such that $u x \in \operatorname{res}(u) \backslash Q$, the set $\delta(u, x)$ is included in $\Sigma^{\leq N}$ and the set $\{\dot{v} r \mid v \in \delta(u, x)\}$ forms a basis of $L H(r)$. In particular, $\delta(u, x)$ contains exactly $d$ elements.

Let $\left(A_{m}\right)_{m \in \mathbb{N}}$ be an enumeration of $\mathcal{S}_{r}$.

Now let $\left(f_{n}\right)_{n \in \mathbb{N}}$ be a family of functions defined as follows: for any integer $n, f_{n}: \operatorname{res}(r) \rightarrow \mathbb{R}, f_{n}(\varepsilon)=1$ and for any $x \in \Sigma$ and $u x \in \operatorname{res}(r)$, $f_{n}(u x)=\frac{|r|\left(u x \Sigma^{\leq n}\right)}{|r|\left(u \Sigma^{\leq n+1}\right)}$ if $|r|\left(u \Sigma^{\leq n}\right) \neq 0$ and $f_{n}(u x)=1$ otherwise. Note that $\lim _{n \rightarrow \infty} f_{n}(u x)=\frac{|r|\left(u x \Sigma^{*}\right)}{|r|\left(u \Sigma^{*}\right)}$.

Let $m \in \mathbb{N}$. For any integer $n$, we define a MA $A_{m, n}$ whose support is equal to $A_{m}=\left\langle\Sigma, Q_{m}, \delta_{m},\{\varepsilon\}, T_{m}\right\rangle$. Let $f: Q_{m} \rightarrow \mathbb{R}$ be defined by $f(u)=f_{n}(u)$. Let $F_{m}=\left\{u x \mid u \in Q_{m}, x \in \Sigma, u x \notin Q_{m}\right\}$ and let $g: F_{m} \times Q_{m} \rightarrow \mathbb{R}$ be defined by $g(u x, v)=0$ if $v \notin \delta_{m}(u, x)$ and $\dot{\overline{u x}} r=\sum_{v \in Q} g(u x, v) \frac{\bar{f}(u)}{\bar{f}(v)} \dot{v} r$ : note that $g$ is completely determined by $A_{m}, f_{n}$ and $r$ since for any $u x \in F_{m},\{\dot{v} r \mid v \in \delta(u, x)\}$ forms a basis of $L H(r)$. We let $A_{m, n}=A\left(\Sigma, Q_{m}, f, g, r\right)$.

Now, consider the following algorithm:

- enumerate $(m, n, k) \in \mathbb{N}$

- for each tuple $(m, n, k)$, build the MA $A_{m, n}=\left\langle\Sigma, Q_{m}, \varphi_{m, n}, \iota_{m, n}, \tau_{m, n}\right\rangle$

- if $\operatorname{Max}_{q \in Q_{m}}\left\{\left|\varphi_{m, n}\right|\left(q, \Sigma^{k}, Q_{m}\right)\right\}<1$, halts.

If $r$ is absolutely convergent, from Theorem 1, there exists a prefixial MA $A=\left\langle\Sigma, Q_{m}, \varphi, \iota, \tau\right\rangle$ that computes $r$, whose support is $A_{m}$ for some integer $m$ and such that $\operatorname{Max}_{q \in Q_{m}}\left\{\varphi\left(q, \Sigma^{k}, Q_{m}\right)\right\}<\rho$ for some integer $k$ and some $\rho<1$. Since $\operatorname{Max}_{q \in Q_{m}}\left\{\varphi\left(q, \Sigma^{k}, Q_{m}\right)\right\}$ is a continuous function in the parameters of $A$, and since $\lim _{n \rightarrow \infty} \varphi_{m, n}\left(q, x, q^{\prime}\right)=\varphi\left(q, x, q^{\prime}\right)$ for any states $q, q^{\prime}$ and any letter $x$, for any $\rho<\rho^{\prime}<1$, there exists an integer $N$ such that $\operatorname{Max}_{q \in Q_{m}}\left\{\varphi_{m, n}\left(q, \Sigma^{k}, Q_{m}\right)\right\}<\rho^{\prime}$ for any integer $n \geq N$. Hence, the algorithm halts on input $r$.

Clearly, from Lemma 3 , since any MA $A_{m, n}$ computes $r$, the algorithm does not halt if $r$ is not absolutely convergent.

\section{Approximation and $L_{1}$-Distance}

To our knowledge, the sum $|r|\left(\Sigma^{*}\right)$ cannot be exactly computed. Nevertheless, it is possible to estimate it by a lower bound since for any integer $n,|r|\left(\Sigma^{\leq n}\right) \leq$ 
$|r|\left(\Sigma^{*}\right)$ and $\lim _{n \rightarrow \infty}|r|\left(\Sigma^{\leq n}\right)=|r|\left(\Sigma^{*}\right)$. We will now define upper bounds of $|r|\left(\Sigma^{*}\right)$ which tend to $|r|\left(\Sigma^{*}\right)$. Hence, it is possible to bound the error made by the approximation to any accuracy rate.

We first give a variation of Theorem 1 .

Lemma 5. Let $r \in \mathcal{A}^{\text {rat }}(\Sigma)$. For any $u \in \operatorname{res}(r)$ and any $x \in \Sigma$, let $f_{n}(u x)=$ $\frac{|r|\left(u x \Sigma^{\leq n}\right)}{|r|\left(u \Sigma^{\leq n+1}\right)}$ if $|r|\left(u \Sigma^{\leq n+1}\right) \neq 0$ and $f_{n}(u x)=1$ otherwise: $\lim _{n \rightarrow \infty} f_{n}(u x)=$ $f(u x)=\frac{|r|\left(u x \Sigma^{*}\right)}{|r|\left(u \Sigma^{*}\right)}$. Let $T>|r|\left(\Sigma^{*}\right)$.

For any integers $N$ and $k$, let $D: \Sigma^{N+k+1} \rightarrow 2^{\Sigma^{\leq N}}$ be such that for any $u x \in$ $\Sigma^{N+k+1},\{\dot{v} r \mid v \in D(u x)\}$ forms a basis of $L H(r)$. In particular, $|D(u x)|=d=$ $\operatorname{dim}(L H(r))$. Given $N, k, D$ and $f_{n}$, there exists a unique function $g: \Sigma^{N+k+1} \times$ $\Sigma^{\leq N} \rightarrow \mathbb{R}$ such that $g(u x, v)=0$ if $v \notin D(u x)$ and such that $A\left(\Sigma, Q, f_{n}, g, r\right)$ computes $r$ (see Proposition 1). Let us denote it by $A\left(N, k, D, f_{n}\right)$.

Now given $\rho<1$, there exists $k_{0}, N_{0}$ such that $\forall N>N_{0}, \forall k>k_{0}$ there exists $n$ and $D$ such that $A=A\left(N, k, D, f_{n}\right)$ satisfies:

$-\varphi_{|A|}\left(u, \Sigma^{2 k}, Q\right)<\rho$

- $\forall u \in \Sigma^{N+k},|\tau(u)|<T$

Proof. By considering the proof of Theorem 1, it can be proved that there exists integers $N_{0}$ and $k_{0}$ such that $\forall N>N_{0}, \forall k>k_{0}$ there exists $D: \Sigma^{N+k+1} \rightarrow$ $2^{\Sigma^{\leq N}}$ such that $A=A(N, k, D, f)$ satisfies $\varphi_{|A|}\left(u, \Sigma^{2 k}, Q\right)<\rho / 2$ and $\forall u \in$ $\Sigma^{N+k},|\tau(u)| \leq|r|\left(\Sigma^{*}\right)$. Since $\varphi_{\left|A\left(N, k, D, f_{n}\right)\right|}\left(u, \Sigma^{2 k}, Q\right)$ and $\left|\tau_{\left|A\left(N, k, D, f_{n}\right)\right|}(u)\right|$ are continuous relatively to the transition coefficients, there exists an integer $n$ such that the conclusion holds.

Proposition 3. Let $r \in \mathcal{A}^{\text {rat }}(\Sigma)$. Let $\mathbb{A}=\left\{A\left(N, k, D, f_{n}\right) / N, k, n \in \mathbb{N}, D \in\right.$ $\left.\mathcal{P}\left(\Sigma^{N}\right)\right\}$. Then $\inf \left(r_{|A|}\left(\Sigma^{*}\right)\right)_{A \in \mathbb{A}}=|r|\left(\Sigma^{*}\right)$.

Proof. Let $N_{0}$ and $k_{0}$ be such as in Lemma 5 . We define a sequence $\left(A_{z}\right)_{z \in \mathbb{N}}$ of automata:

$-N_{z}=N_{0}+z, k_{z}=k_{0}$

- $\forall z, n_{z}$ and $D_{z}$ are such as in Lemma $5, A_{z}=A\left(N_{z}, k_{z}, D_{z}, f_{n_{z}}\right)$.

As $N_{z}$ grows, $r_{\left|A_{z}\right|}$ converges pointwisely to $|r|$. We have $\left\|\tau_{z}\right\|_{\infty}<T$ by construction. We have $R=\sup _{h<2 k_{z}}\left(\left|\varphi_{z}\right|\left(\varepsilon, \Sigma^{2 k_{z}}, Q\right)\right)<1$, as $\left.\left|\varphi_{z}\right|(u, \Sigma, Q)\right)<1$ for $u \in \Sigma^{<N_{z}+k_{z}}$. Applying Lemma 4, we have $r_{\left|A_{z}\right|}\left(\Sigma^{>n}\right)<T \rho^{n / 2 k_{0}-1}$ and therefore $|r|\left(\Sigma^{>n}\right)<T \rho^{n / 2 k_{0}-1}$. Now, $\left|r_{\left|A_{z}\right|}\left(\Sigma^{*}\right)-\right| r\left|\left(\Sigma^{*}\right)\right| \leq \sum_{w \in \Sigma^{*}} \mid r_{\left|A_{z}\right|}(w)-$ $|r|(w)\left|\leq \sum_{w \in \Sigma \leq Z_{1}}\right| r_{\left|A_{z}\right|}|(w)-| r|(w)|+\sum_{w \in \Sigma>Z_{1}} r_{\left|A_{z}\right|}(w)+\sum_{w \in \Sigma>Z_{1}}|r|(w)$. For $\epsilon>0$, one can find $Z_{1}$ such that both $\sum_{w \in \Sigma^{>Z_{1}}} r_{\left|A_{z}\right|}(w)<\epsilon / 3$ and $\sum_{w \in \Sigma^{>} z_{1}}|r|(w)<\epsilon / 3$. Finally, as $r_{\left|A_{z}\right|}$ converges pointwisely to $|r|(w)$, one can find $Z_{2}$ such that $\sum_{w \in \Sigma \leq Z_{1}}\left|r_{\left|A_{z}\right|}(w)-\right| r|(w)|<\epsilon / 3$, and we can conclude.

Proposition 4. Let $A_{Z}$ be an enumeration of the set $\{A(N, k, D, n) / N, k, n \in$ $\left.\mathbb{N}, D \in \mathcal{P}\left(\Sigma^{N}\right)\right\}$. Let $G_{0}=r_{\left|A\left(N_{0}, k_{0}, D_{0}, f_{n_{0}}\right)\right|}\left(\Sigma^{*}\right)$. Let $G_{z}=r_{\left|A_{z}\right|}\left(\Sigma^{*}\right)$ if it exists, and if $G_{z}<G_{z-1}, G_{z}=G_{z-1}$ otherwise. Then $\lim _{z \rightarrow \infty} G_{z}=|r|\left(\Sigma^{*}\right)$. 
Proof. Straightforward from Proposition 3.

Theorem 3. Let $r \in \mathcal{A}^{\text {rat }}(\Sigma)$. For any $\varepsilon>0$, it is possible to compute an estimate $\mid \widehat{r \mid\left(\Sigma^{*}\right)}$ of $|r|\left(\Sigma^{*}\right)$ such that $\left|\widehat{|r|\left(\Sigma^{*}\right)}-\right| r\left|\left(\Sigma^{*}\right)\right|<\varepsilon$. If the $L_{1}$-distance $d$ of two rational series $r$ and $s$ is finite, for any $\varepsilon>0$, it is possible to compute an estimate $\hat{d}$ of $d$ such that $|\hat{d}-d|<\varepsilon$.

Proof. Using Proposition 4, find $G_{z}$ and $n$ such that $G_{z}-|r|\left(\Sigma^{\leq n}\right)<2 \varepsilon$. For the second part, apply the result to $r-s$.

\section{Conclusion}

In this paper, we have proved that it is semi-decidable whether a rational series is absolutely convergent. Then, given an absolutely convergent rational series $r$, we have provided an algorithmic way to estimate the sum $|r|\left(\Sigma^{*}\right)$ to any accuracy rate. We do not know whether $\mathcal{A}^{\text {rat }}(\Sigma)$ is decidable. We conjecture that it should be decided that a rational series $r$ is not absolutely convergent when $\rho_{|r|}>1$ but the case $\rho_{|r|}=1$ is likely to be difficult. We intend to study the links between the spectral radius we have defined and the joint or generalized spectral radii. Finally, we are currently looking for more efficient algorithms and heuristics than those we have described to approximate the L1 distance of two rational series, even if there is no hope to find efficient algorithms in the worst case.

\section{References}

1. Berstel, J., Reutenauer, C.: Noncommutative Rational Series With Applications. to be published in Cambridge University Press (2008)

2. Salomaa, A., Soittola, M.: Automata: Theoretic Aspects of Formal Power Series. Springer-Verlag (1978)

3. Denis, F., Esposito, Y.: On rational stochastic languages. Fundamenta Informaticae 86(1-2) (2008) 41-77

4. Denis, F., Esposito, Y., Habrard, A.: Learning rational stochastic languages. In: Proceedings of the 19th Annual Conference on Learning Theory (COLT'06). Volume 4005 of LNCS., Springer (2006) 274-288

5. Lyngs $\varnothing$ R.B., Pedersen, C.N.S.: The consensus string problem and the complexity of comparing hidden markov models. J. Comput. Syst. Sci. 65(3) (2002) 545-569

6. Cortes, C., Mohri, M., Rastogi, A.: On the computation of some standard distances between probabilistic automata. In Ibarra, O.H., Yen, H.C., eds.: CIAA. Volume 4094 of Lecture Notes in Computer Science., Springer (2006) 137-149

7. Cortes, C., Mohri, M., Rastogi, A.: Lp distance and equivalence of probabilistic automata. Int. J. Found. Comput. Sci. 18(4) (2007) 761-779

8. Theys, J.: Joint Spectral Radius : theory and approximations. PhD thesis, UCL Université Catholique de Louvain, Louvain-la-Neuve, Belgium (2005) 